\title{
On The Double-Vacua Duality of Multi-Scalar Higgs and NGB-Dual Higgses in Scherk-Schwarz Breaking of 5-dimensional SU(6) Symmetry
}

\author{
Jusak Sali Kosasih ${ }^{1,2}$, Andreas Hartanto ${ }^{1,2}$, Laksana Tri Handoko ${ }^{3,4}$, \\ \& Freddy Permana Zen ${ }^{1,2}$ \\ ${ }^{1}$ Indonesian Center for Theoretical and Mathematical Physics \\ Institut Teknologi Bandung, Jalan Ganesha 10, Bandung 40132, Indonesia \\ ${ }^{2}$ Theoretical Physics Laboratory, THEPI Research Division, \\ Institut Teknologi Bandung, Jalan Ganesha 10, Bandung 40132, Indonesia \\ ${ }^{3}$ Department of Physics, University of Indonesia, Depok 16424, West Java, Indonesia \\ ${ }^{4}$ Theoretical and Computational Physics Group, Research Center for Physics, \\ Indonesian Institute of Science (LIPI), Puspiptek, Tangerang 15314, Banten, Indonesia, \\ Email: jusak@fi.itb.ac.id
}

\begin{abstract}
A special condition of Scherk-Schwarz and $S^{1} / Z_{2}$ orbifold breaking brings about both a weakly-coupled $\mathrm{SU}(6)$ baby Higgs and a strongly-coupled will-be simplest little Higgs scalar in the near-brane of $\mathrm{SU}(3) \times \mathrm{SU}(3) \times \mathrm{U}(1)$. The latter produces $\mathrm{SU}(3)$ VEVs and simplest little-like Higgs after triplet-triplet splitting and, under quadratic-based and non-quadratic-based Coleman-Weinberg potential, the simplest little-like Higgs yields exotic Higgses, scalar-pair and 3scalar Higgses in the so-called one-by-one and collective breakings. A generalized non-quadratic-based Coleman-Weinberg potential utilizing a NGB-like scalar produces NGB-dual Higgses with a squared mass relevant to the components of a 3-scalar Higgs that further create a duality of 3-scalar Higgs and NGB-dual Higgses. This is due to a double-vacua property such that each vacuum responds equally to the shifts happening at either non-zero or zero-VEV vacuum.
\end{abstract}

Keywords: Orbifold; Scherk-Schwarz breaking; little Higgs; Standard Model; Coleman-Weinberg potential; Nambu-Goldstone boson.

\section{Introduction}

There are various extensions of the Standard Model, including theories with extra dimensions. One of these approaches is to define 5D SU(6) gauge symmetry and, based on AdS/CFT correspondence, its relevant SU(6) global symmetry [1], which breaks after shift symmetry breaking and produces a massive PNB. The scalars come from the fifth component of the 5D gauge bosons [1-4] and/or directly from the bulk [5].

In electroweak theory, the third component of the massless gauge boson shows up as the Nambu-Goldstone boson as additional degree of freedom,

Received June $19^{\text {th }}, 2013$, Revised April 12 ${ }^{\text {th }}, 2014$, Accepted for publication May 30 2014.

Copyright (C) 2014 Published by ITB Journal Publisher, ISSN: 2337-5760, DOI: 10.5614/j.math.fund.sci.2014.46.2.3 
transforming the gauge boson into a massive state. The third component of isospin is used to construct a holonomy in $\mathrm{SU}(2)_{\mathrm{L}} \times \mathrm{U}(1)_{\mathrm{Y}}$, which is extended to $5 \mathrm{D} \mathrm{SU}(2) \times \mathrm{U}(1)$ by replacing the $3 \mathrm{rd}$ isospin component with the fifth component of the 5D gauge boson as a phase factor of the holonomy [6]. The symmetry is broken if the fifth component has non-zero VEV.

It is clear that through the $3 \mathrm{rd}$ isospin component, a correspondence between the Nambu-Goldstone boson and the broken fifth component of the 5D gauge boson is established as the basis for constructing a generalized Coleman-Weinberg potential.

In [7], special conditions of Scherk-Schwarz mechanisms are utilized to resolve the problem of breaking the SU(6) GUT. The trivial and non-trivial breaking patterns are simultaneously realized by compactification on the $S^{1} / Z_{2}$ orbifold in the 5-dimensional SU(6) model, where only the (pseudo) non-trivial condition demands the existence of scalar bosons. This condition facilitates the periodic 5D scalar [1,3,8-18] with extra-dimensional global symmetry in a small extra dimension, the so-called near-brane area [1,7]. In the near-brane area, the first symmetry breaking of $5 \mathrm{D} \mathrm{SU}(6) \rightarrow 4 \mathrm{D}$ SU(6) is triggered by the ScherkSchwarz mechanism and followed by trivial and pseudo non-trivial orbifold breaking [4-8] to produce an SU(6)-origin little (baby) Higgs scalar as the origin of SU(6) will-be simplest little Higgs and SU(6) baby Higgs scalars successively [7]. The second symmetry breaking 4D SU(6) $\rightarrow 4 \mathrm{D}$ SU(3) $\times$ $\mathrm{SU}(3) \times \mathrm{U}(1)$ is induced by a SU(6) little-like Higgs through orbifold-based field redefinition and the broken shift symmetry in the lower-near-brane $[1,4,7]$. The VEVs are obtained from two Scherk-Schwarz parameters [2,6,19-20].

In this paper, the 5D model is constructed based on [7] together with ScherkSchwarz and $S^{1} / Z_{2}$ orbifold breaking. The model makes use of a near-brane Coleman-Weinberg potential and its generalized version on which the quantum route of the SU(6) will-be simplest little Higgs scalar will be based. One can immediately predict the birth of the simplest little Higgs from the SU(6) will-be simplest little Higgs based on pseudo non-trivial breaking where $\mathrm{SU}(3) \times$ $\mathrm{SU}(3) \times \mathrm{U}(1)$ symmetry shows up. Here, the emerging of one-by-one and collective breaking potentials and Higgses are discussed. Moreover an NGBlike scalar is established and a generalized Coleman-Weinberg potential is constructed, which, after some simplification, produces the components of the above multi-scalar Higgs. Before closing the paper, a brief discussion on the duality of the two types of Higgses in a double vacua is given. 


\section{The 5D SU(6) Breaking Formalism}

\subsection{The Scherk-Schwarz and Orbifold Breaking}

In the 5D theory, compactified on the $S^{1} / Z_{2}$ orbifold with the Scherk-Schwarz twist, the field obeys,

$$
\phi(x, y+2 \pi R)=e^{2 i \pi \omega Q} \phi(x, y)
$$

where $R$ is the compactification radius. The symmetry generated by $Q$ is broken by the 5D kinetic term, while the one generated by $Q^{\prime}$ is unbroken if $Q^{\prime}(Q)$ satisfies the following (anti)commutative relation [6],

$$
\left\{\omega \mathrm{Q}, \mathrm{Z}_{2}\right\}=\omega\left\{\mathrm{Q}, \mathrm{Z}_{2}\right\}=0, \quad\left[\mathrm{Q}^{\prime}, \mathrm{Z}_{2}\right]=0 .
$$

We now apply the preceding discussion on the $S^{1} / Z_{2}$ orbifold to $\operatorname{SU}(6)[5,6] . Z_{2}$ for $\mathrm{SU}(6)$ can be constructed as follows,

$$
Z_{2}=\operatorname{diag}\left(\left(\begin{array}{ll}
1 & 0 \\
0 & 1
\end{array}\right),\left(\begin{array}{rr}
1 & 0 \\
0 & -1
\end{array}\right),\left(\begin{array}{rr}
-1 & 0 \\
0 & -1
\end{array}\right)\right)
$$

This form satisfies the boundary conditions of the $S^{1} / Z_{2}$ orbifold suitable to realize the symmetry breaking $\mathrm{SU}(6) \rightarrow \mathrm{SU}(3) \times \mathrm{SU}(3) \times \mathrm{U}(1)$ in a non-trivial pattern. A special condition emerges from the anti-commutator in Eq. (2) if $\omega \neq$ $0, Z_{2}$ as in Eq. (3), and $Q=0$, which defines a pseudo non-trivial pattern with $5 \mathrm{D} \mathrm{SU}(6) \rightarrow 4 \mathrm{D} \mathrm{SU}(6)$. This means dimensional deconstruction does not bring gauge symmetry breaking. The same result is obtained if one takes $Z_{2}=U$ in the commutator in Eq. (2), where $U=Z_{2}^{(1)} Z_{2}^{(2)}=I_{6}$, as shown by Eq. (3), which demands all $\mathrm{SU}(6)$ generators for $Q^{\prime}$ to be unbroken, hence defining a trivial pattern. Both patterns facilitate SU(6) little-like bosons in massless state.

\subsection{The 5D-Model and SU(6) Would-be Baby (Little) Higgs}

We adopt the 5D-model with 4D particles in 2 branes and 5D gauge bosons with scalar bosons in the bulk. The two branes correspond to the fixed points $y$ $=0$ and $y=\pi R$ of the $S^{1} / Z_{2}$ orbifold.

With $\widetilde{\Phi}$ in the fundamental representation of SU(6), the bulk scalar boson $\Phi(x, y)$ can be expressed as periodic scalar field $\widetilde{\Phi}$ via the relation [6],

$$
\Phi(\mathrm{x}, \mathrm{y})=\mathrm{e}^{\mathrm{i} \omega \mathrm{Q}_{\mathrm{v}} \mathrm{y} / \mathrm{R}} \widetilde{\Phi}(\mathrm{x}, \mathrm{y}),
$$

with $Q_{v}$ is the broken generators of $\mathrm{SU}(6)$ in the VEV direction $[4,6]$. 
Under the gauge-Higgs Unification Principle [7,8], $\widetilde{\Phi}$ has Kaluza-Klein (KK) expansion forms for the even $(y=0)$ and odd $(y=\pi R)$ scalars due to parity splitting under the dimensional deconstruction, as follows, resp.

$$
\begin{aligned}
& \widetilde{\Phi}_{+}^{(i)}=\frac{1}{\sqrt{\pi R}}\left[\widetilde{\Phi}_{+}^{0}(\mathrm{x})\right]_{\mathrm{i}}+\sqrt{\frac{1}{\pi \mathrm{R}}} \sum_{\mathrm{n}=2}^{\infty}\left[\widetilde{\Phi}_{+}^{\mathrm{n}}(\mathrm{x})\right]_{\mathrm{i}} \cos \left(\frac{\mathrm{ny}}{\mathrm{R}}\right), \\
& \widetilde{\Phi}_{-}^{(\mathrm{i})}=\sqrt{\frac{1}{\pi \mathrm{R}}} \sum_{\mathrm{n}=1}^{\infty}\left[\widetilde{\Phi}_{-}^{\mathrm{n}}(\mathrm{x})\right]_{\mathrm{i}} \sin \left(\frac{\mathrm{ny}}{\mathrm{R}}\right),
\end{aligned}
$$

where $\widetilde{\Phi}_{+}^{(i)}, \mathrm{i}=1,2$ are the original bulk scalars.

At the orbifold singular points, which are 4D and have twist factors $T_{g}=+1$ for $y=0$ and $T_{g}=-1$ for $y=\pi R$, two non-zero VEVs can be assigned at one fixed point, without losing any generality [7],

$$
\mathrm{v}=\frac{1}{\sqrt{\pi \mathrm{R}}}\left(\begin{array}{llllll}
0 & 0 & \mathrm{f}_{1} & 0 & 0 & 0
\end{array}\right)^{\mathrm{T}}, \mathrm{v}^{\prime}=\frac{1}{\sqrt{\pi \mathrm{R}}}\left(\begin{array}{llllll}
0 & 0 & 0 & 0 & 0 & \mathrm{f}_{2}
\end{array}\right)^{\mathrm{T}}, \text { for } \mathrm{y}=0 .
$$

The parametrization of the SU(6) would-be baby (little) Higgs is governed by the number of scalar doublets that can be arranged in a $6 \times 6$ matrix. Thus, it depends on the number of generated NGBs through the condition [7],

$$
\mathrm{a}^{\prime}{ }_{\mathrm{jk}} \widetilde{\Phi}_{\mathrm{k}} \neq 0, \quad\left(\widetilde{\Phi}_{\mathrm{k}}\right)_{01}=\mathrm{v}, \quad\left(\widetilde{\Phi}_{\mathrm{k}}\right)_{02}=\mathrm{v}^{\prime},
$$

with $a^{\prime}=1, \ldots, 35$. Eq. (8) gives a total of 22 free NGBs. Finally, one has 8 scalar bosons that create 4 scalar doublets to be assigned as the $\mathrm{SU}(6)$ would-be baby (little) Higgs as follows [7],

$$
\theta=\frac{1}{\mathrm{f}}\left(\begin{array}{ccc}
(0)_{3 \times 3} & \left(\begin{array}{cc}
(0)_{2 \times 2} & (\mathrm{~h})_{2 \times 1} \\
\left(\mathrm{~h}^{\prime \dagger}\right)_{1 \times 2} & 0
\end{array}\right) \\
(0)_{2 \times 2} & \left(\mathrm{~h}^{\prime}\right)_{2 \times 1} \\
\left(\mathrm{~h}^{\dagger}\right)_{1 \times 2} & 0
\end{array}\right),
$$

where $\mathrm{f}^{2}=\mathrm{f}_{1}^{2}+\mathrm{f}_{2}^{2}$. The scalar doublets $h$ and $h$ ' are would-be SM Higgses.

The exponential forms of the IR near-brane 4D SU(6) scalars follow directly from Eq. (5), Eq. (7) and Eq. (9)

$$
\widetilde{\Phi}_{+}^{(1)}\left[\widetilde{\Phi}_{+}^{(2)}\right]=\mathrm{e}^{\frac{\mathrm{if} f_{1}}{\mathrm{ff}_{1}}\left[\frac{-\mathrm{if}_{1}}{\mathrm{ff}_{2}}\right]\left(\begin{array}{cc}
(0)_{3 \times 3} & \left(\begin{array}{cc}
(0)_{2 \times 2} & \mathrm{~h} \\
\mathrm{~h}^{\prime \dagger} & 0
\end{array}\right) \\
\left(\begin{array}{cc}
(0)_{2 \times 2} & \mathrm{~h}^{\prime} \\
\mathrm{h}^{\dagger} & 0
\end{array}\right) & (0)_{3 \times 3}
\end{array}\right) \mathrm{v}\left[\mathrm{v}^{\prime}\right] .}
$$

This is similar to the principle of PNB parametrization in the simplest little Higgs $[3,13,15]$, and they are named SU(6) would-be baby (little) Higgses. 


\subsection{Near-Brane Coleman-Weinberg Potential and Baby Higgs}

Accordingly, the Lagrangian can be written as

$$
\mathrm{L}_{5}^{\mathrm{SU}(6)}=\mathrm{D}^{\mathrm{M}} \Phi^{\dagger} \mathrm{D}_{\mathrm{M}} \Phi, \quad \mathrm{M}=(\mu, \mathrm{y})
$$

where $\Phi=\left(\Phi_{1}, \Phi_{2}, \Phi_{3}, \Phi_{4}, \Phi_{5}, \Phi_{6}\right)^{\mathrm{T}} \equiv\left[\Phi_{\mathrm{k}}\right], \mathrm{k}=1,2, \cdots, 6$ is a scalar boson sextet of SU(6), and scalar field $\Phi$ is re-expressed as periodic scalar field $\widetilde{\Phi}$ via the following relation [6], which is identical to Eq.(4),

$$
\Phi(\mathrm{x}, \mathrm{y})=\mathrm{e}^{\mathrm{i} \mathrm{Q}_{\mathrm{v}} \alpha} \widetilde{\Phi}(\mathrm{x}, \mathrm{y}), \quad \alpha=\frac{\omega \mathrm{y}}{\mathrm{R}} .
$$

Defining $D_{\mu}$ and $D_{y}$ as $4 \mathrm{D}$ and fifth-dimension covariant derivatives respectively, with $T^{a}=\lambda^{a} / 2=T_{a}$, while $g_{5}$ is the 5D coupling constant,

$$
\begin{gathered}
D_{\mu}=\partial_{\mu}-i g_{5} A_{\mu}^{a} T_{a}, \quad D^{\mu}=\partial^{\mu}+i g_{5} A_{a}^{\mu} T^{a} \\
\mathrm{D}_{\mathrm{y}}=\partial_{\mathrm{y}}+i g_{5} \mathrm{~A}_{\mathrm{y}}^{\mathrm{a}} \mathrm{T}_{\mathrm{a}}, \quad \mathrm{D}^{\mathrm{y}}=\partial^{\mathrm{y}}-\mathrm{ig}_{5} \mathrm{~A}_{\mathrm{a}}^{\mathrm{y}} \mathrm{T}^{\mathrm{a}},
\end{gathered}
$$

the bulk, $\mathrm{L}_{5}^{\mathrm{SU}(6)}=\mathrm{L}_{\mu}^{\text {brane }}+\mathrm{L}_{(\theta \mathrm{y}), \mathrm{y}}^{\text {near-brane }}+\mathrm{L}_{\mathrm{y}}^{\mathrm{SU}(6)}$, can be separated from the $4 \mathrm{D}$ near-brane Lagrangian, which is just in between the brane and the bulk.

Setting $Q_{v}=0$ for the SU(6) upper-near-brane (thus eliminating the Lorentz invariant-violating term), the Lagrangians, after Scherk-Schwarz but prior to orbifold breaking, can be expressed as follows,

$$
\begin{aligned}
& \mathrm{L}_{\mu}^{\text {brane }}=\mathrm{D}^{\mu} \widetilde{\Phi}^{\dagger} \mathrm{D}_{\mu} \widetilde{\Phi} \\
& \mathrm{L}_{\mathrm{y}}^{\mathrm{SU}(6)}=\mathrm{D}^{\mathrm{y}} \widetilde{\Phi}^{\dagger} \mathrm{D}_{\mathrm{y}} \widetilde{\Phi}+\mathrm{ig}_{5}\left(\mathrm{~A}_{\mathrm{y}}^{\mathrm{a}} \mathrm{T}_{\mathrm{a}} \widetilde{\Phi} \mathrm{D}^{\mathrm{y}} \widetilde{\Phi}^{\dagger}-\mathrm{A}_{\mathrm{a}}^{\mathrm{y}} \mathrm{T}^{\mathrm{a}} \widetilde{\Phi}^{\dagger} \mathrm{D}_{\mathrm{y}} \widetilde{\Phi}\right) .
\end{aligned}
$$

For $\mathrm{L}_{(\theta \mathrm{y}), \mathrm{y}}^{\text {nean-bre }}$ two cases are based on shift-symmetry-breaking parameter $(\theta \alpha)$ (or $(\theta y)$ due to $\alpha=\omega y / R$ ) as follows: in the upper-near-brane where shift symmetry is intact and $\theta$ is small, $(y \sim 0,(\theta y) \rightarrow 0), D^{\mu} \widetilde{\Phi}^{\dagger}=D_{\mu} \widetilde{\Phi}=0$ due to $\partial^{\mu} \theta\left(\right.$ or $\left.\partial_{\mu} \theta\right) \rightarrow 0$, while in the lower-near-brane, where shift symmetry is broken and $(\theta y)$-term and $\theta$ are significant, one finds $(y \sim 0,(\theta y) \rightarrow \mu \neq 0)$, $D^{\mu} \widetilde{\Phi}^{\dagger} \neq D_{\mu} \widetilde{\Phi} \neq 0$ due to the significant value of $\partial^{\mu} \theta\left(\partial_{\mu} \theta\right)$. Noting $\mathcal{L}_{(\theta y), y}^{\text {near-brane }}=\mathcal{L}_{\mu}^{\text {brane }}$ and $\mathcal{L}_{(\theta y), y}^{\text {near-brane }}=\mathcal{L}_{y}^{\text {near-brane }}$, one gets for the upper-nearbrane,

$$
\mathrm{L}_{\mathrm{y}}^{\text {near-brane }}=\delta(\mathrm{y})\left\{\frac{1}{2} \mathrm{~g}_{5}^{2} \widetilde{\Phi}^{\dagger} \mathrm{A}_{\mathrm{a}}^{\mathrm{y}} \mathrm{A}_{\mathrm{y}}^{\mathrm{a}} \widetilde{\Phi}\right\},
$$

and for the lower-near-brane, for the reason which will be clear after Eq. (19),

$$
\mathrm{L}_{\mu}^{\text {near-brane }}=\mathrm{V}_{\mu}^{(6)},
$$


where $V_{\mu}^{(6)}$ will turn out to be $V_{\mu P}^{(6)}$ in Eq. (26).

Eq. (14) reflects the condition in the brane $(y=0, \mu)$, while Eq. (15) is for the far-distant out-of-brane condition $(y>0, \mu)$.

For the upper-near-brane area Neumann the boundary condition dictates $\mathrm{D}^{\mathrm{y}} \widetilde{\Phi}^{\dagger}=\mathrm{D}_{\mathrm{y}} \widetilde{\Phi}=0$ and, based on the property of extra-dimensional dominance, makes Eqs. (14) and (15) zero. This shows that Eq. (16) is the only upper-nearbrane equation with $\delta(y) \neq 0$ for $y \sim 0$ and under both trivial and pseudo nontrivial breaking conditions, $Q_{v}=0$, we obtain $\left(\widetilde{\Phi}^{(i)}=\widetilde{\Phi}_{+}^{(i)}(x)\right.$ and $\widetilde{\Phi}^{(i) \dagger}=$ $\left.\widetilde{\Phi}_{+}^{(i) \dagger}(x)\right)$

$$
\mathrm{L}_{\mathrm{y}}^{\text {near-brane }}=\frac{1}{2} \mathrm{~g}_{5}^{2}\left(\widetilde{\Phi}^{(\mathrm{i}) \dagger} \mathrm{A}_{\mathrm{a}}^{\mathrm{y}}\right)\left(\mathrm{A}_{\mathrm{y}}^{\mathrm{a}} \widetilde{\Phi}^{(\mathrm{i})}\right) i=1,2 .
$$

In this upper-near-brane bulk (extradimensionally-dominated space) under the provision of pseudo non-trivial orbifold breaking, by which $A_{\hat{a}}^{\mathrm{y}} \mathrm{T}^{\hat{\mathrm{a}}}$ and $A_{y}^{\hat{a}} T_{\hat{a}}$ produce a upper-near-brane scalar due to gauge-scalar unification $[2,4]$, one has the relations (sextet out of $2 \times 9$ broken $A_{\hat{a}}^{\mathrm{y}}$ and $A_{\mathrm{y}}^{\hat{\mathrm{a}}}$ ) as follows

$$
A_{\hat{a}}^{\mathrm{y}} T^{\hat{a}} \supset \widetilde{\Phi}^{(j)}, \quad A_{\mathrm{y}}^{\hat{a}} T_{\hat{a}} \supset \widetilde{\Phi}^{(j) \dagger} .
$$

$\widetilde{\Phi}^{(j)}$ (or $\widetilde{\Phi}^{(j) \dagger}$ ) is a diagonal $3 \times 3$ sub-matrix component of the $6 \times 6$ matrix of $A_{\hat{a}}^{y} T^{\hat{a}}$ (or $A_{y}^{\hat{a}} T_{\hat{a}}$ ) due to hermitian conjugacy and the condition $D^{\mu} \widetilde{\Phi}^{\dagger}=$ $D^{\mu} A_{y}^{\hat{a}} T_{\widehat{a}}=0, D_{\mu} \widetilde{\Phi}=D_{\mu} A_{\widehat{a}}^{y} T^{\hat{a}}=0$. In the lower-near-brane $\mathrm{D}^{\mu} \widetilde{\Phi}^{\dagger} \neq \mathrm{D}_{\mu} \widetilde{\Phi} \neq 0$ due to the significant $\partial^{\mu} \theta\left(\partial_{\mu} \theta\right)$ because of the dominant $4 \mathrm{D}$ property. Eq. (18) provides the quartic term with $i$ index to label the original scalar boson in Eq. (18), which, using $\widetilde{\Phi}^{(j)}$ as diagonal component of $A_{\widehat{a}}^{\mathrm{y}} \mathrm{T}^{\hat{\mathrm{a}}}$ and $\lambda_{\mathrm{y}}^{(6)}=\mathrm{g}_{5}^{2}$, can be rewritten as

$$
\mathrm{V}_{\mathrm{y}}^{(6)}=\lambda_{\mathrm{y}}^{(6)}\left(\widetilde{\Phi}^{(\mathrm{i}) \dagger} \widetilde{\Phi}^{(\mathrm{j})}\right)\left(\widetilde{\Phi}^{(\mathrm{j}) \dagger} \widetilde{\Phi}^{(\mathrm{i})}\right) .
$$

This scalar lives below energy scale $\Lambda_{(6)}^{\mathrm{NP}}$ and can be represented by $\mathrm{SU}(6)$ baby Higgses that are defined by zero mode approximation in the lowest order perturbation and can be written as ( $P$ : perturbative),

$$
\widetilde{\Phi}_{+, \mathrm{P}}^{(1)}(\mathrm{x})=\mathrm{v}\left(1+\frac{\mathrm{if}_{2}}{\mathrm{f}_{1}} \theta(\mathrm{x})\right), \widetilde{\Phi}_{+, \mathrm{P}}^{(2)}(\mathrm{x})=\mathrm{v}^{\prime}\left(1-\frac{\mathrm{if}_{1}}{\mathrm{f}_{2}} \theta(\mathrm{x})\right) .
$$

Eq. (21) brings us to the orbifold-based field redefinition as follows,

$$
\widetilde{\Phi}_{+, \mathrm{P}}^{(1)^{\prime}}(\mathrm{x})=\widetilde{\Phi}_{+, \mathrm{P}}^{(1)}(\mathrm{x})-\mathrm{v}+\mathrm{v}^{\prime}, \widetilde{\Phi}_{+, \mathrm{P}}^{(2){ }^{\prime}}(\mathrm{x})=\widetilde{\Phi}_{+, \mathrm{P}}^{(2)}(\mathrm{x})-\mathrm{v}^{\prime}+\mathrm{v} .
$$




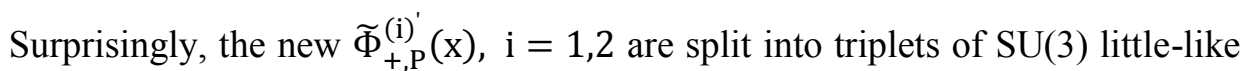
Higgses in accordance to

$$
\widetilde{\Phi}_{+, \mathrm{P}}^{(1)^{\prime}}(\mathrm{x})=\left(\begin{array}{c}
0_{3 \times 1} \\
\phi_{\mathrm{P}}^{(1)}
\end{array}\right), \quad \widetilde{\Phi}_{+, \mathrm{P}}^{(2)^{\prime}}(\mathrm{x})=\left(\begin{array}{c}
\phi_{\mathrm{P}}^{(2)} \\
0_{3 \times 1}
\end{array}\right),
$$

where, with $\Delta \mathrm{f}=\mathrm{f}_{2}-\mathrm{f}_{1}, \mathrm{SU}(3)$ little-like Higgs triplets are defined as

$$
\begin{aligned}
& \phi_{\mathrm{P}}^{(1)}=\frac{1}{\sqrt{\pi \mathrm{R}}}\left[\left\{\left(1+\frac{\Delta \mathrm{f}}{\mathrm{f}_{1}}\right)+\frac{i \mathrm{f}_{2}}{\mathrm{f}_{1} \mathrm{f}}\left(\begin{array}{cc}
(0)_{2 \times 2} & \mathrm{~h}^{\prime} \\
\mathrm{h}^{\dagger} & 0
\end{array}\right)\right\}\left(\begin{array}{c}
0 \\
0 \\
\mathrm{f}_{1}
\end{array}\right)\right], \\
& \phi_{\mathrm{P}}^{(2)}=\frac{1}{\sqrt{\pi \mathrm{R}}}\left[\left\{\left(1-\frac{\Delta \mathrm{f}}{\mathrm{f}_{2}}\right)-\frac{\mathrm{i \textrm {f } _ { 1 }}}{\mathrm{f}_{2} \mathrm{f}}\left(\begin{array}{cc}
(0)_{2 \times 2} & h \\
\mathrm{~h}^{\prime} & 0
\end{array}\right)\right\}\left(\begin{array}{l}
0 \\
0 \\
\mathrm{f}_{2}
\end{array}\right)\right] .
\end{aligned}
$$

The potential of the SU(6) baby Higgses follows from Eq. (17) and (20) by replacing $\lambda_{y}{ }^{(6)} \rightarrow \lambda_{\mu P}{ }^{(6)}$, for $i, j=1,2$, and $\widetilde{\Phi}_{+, \mathrm{P}}^{(\mathrm{i})^{\prime}} \sim \widetilde{\Phi}_{+, \mathrm{P}}^{(\mathrm{j})^{\prime}}$ for $i \neq j$. From Eq. (23) one finds $\widetilde{\Phi}_{+, \mathrm{P}}^{(\mathrm{i}){ }^{\prime} \dagger} \widetilde{\Phi}_{+, \mathrm{P}}^{(\mathrm{j}){ }^{\prime}}=0$ for $i \neq j$. Therefore Eq. (20) is rewritten as

$$
\mathrm{V}_{\mu \mathrm{P}}^{(6)}=\lambda_{\mu \mathrm{P}}^{(6)}\left\{\left(\widetilde{\Phi}_{+, \mathrm{P}}^{(1)^{\prime} \dagger} \widetilde{\Phi}_{+, \mathrm{P}}^{(1)^{\prime}}\right)^{2}+\left(\widetilde{\Phi}_{+, \mathrm{P}}^{(2)^{\prime} \dagger} \widetilde{\Phi}_{+, \mathrm{P}}^{(2)^{\prime}}\right)^{2}\right\}
$$

which is the sum of potentials of three Higgses [7].

\section{SU(3)-Origin Higgses from SU(6) Near-Brane}

\subsection{The Strongly-Coupled SU(6) will-be Simplest Little Higgs Scalar}

Let us start with the upper-near-brane Lagrangian, which is basically a quartic potential [7] in Eq. (20), where $\lambda_{y}{ }^{(6)}$ is the coupling constant of the upper-nearbrane, while $\widetilde{\Phi}_{+}^{(\mathrm{i})}$ and $\widetilde{\Phi}_{+}^{(\mathrm{j})}$ are the SU(6)-origin would-be little (baby) Higgs bosons as expressed in Eq. (10) [7].

The Lagrangian in the lower-near-brane can be obtained from Eq. (20) by replacing $\lambda_{y}{ }^{(6)} \rightarrow \lambda_{y N P}{ }^{(6)}$ and $\widetilde{\Phi}_{+}^{(\mathrm{i})} \rightarrow \widetilde{\Phi}_{+}^{(\mathrm{i})^{\prime}}, \widetilde{\Phi}_{+}^{(\mathrm{j})} \rightarrow \widetilde{\Phi}_{+}^{(\mathrm{j}){ }^{\prime}}$ as follows,

$$
\mathrm{L}_{\mathrm{y}}^{\text {near-brane }}=\mathrm{V}_{\mathrm{yNP}}^{(6)}=\lambda_{\mathrm{yNP}}^{(6)} \widetilde{\Phi}_{+}^{(\mathrm{i})^{\prime} \dagger} \widetilde{\Phi}_{+}^{(\mathrm{j})^{\prime}} \widetilde{\Phi}_{+}^{(\mathrm{j})^{\prime} \dagger} \widetilde{\Phi}_{+}^{(\mathrm{i})^{\prime}},
$$

where $\lambda_{y N P}{ }^{(6)}$ is the coupling constant of the lower-near-brane. $\widetilde{\Phi}_{+}^{(\mathrm{i})}, \widetilde{\Phi}_{+}^{(\mathrm{j}){ }^{\prime}}$ are the SU(6) will-be simplest little Higgs bosons, which can be written as [7], 


$$
\widetilde{\Phi}_{+}^{(1)^{\prime}}=\left(\begin{array}{l}
\phi_{0}^{(1)} \\
\phi^{(1)}
\end{array}\right), \quad \widetilde{\Phi}_{+}^{(2)^{\prime}}=\left(\begin{array}{c}
\phi^{(2)} \\
\phi_{0}^{(2)}
\end{array}\right),
$$

with the following SU(3) VEV triplets,

$$
\phi_{0}^{(1)}\left[\phi_{0}^{(2)}\right]=\frac{1}{\sqrt{\pi R}}\left(\begin{array}{c}
0 \\
0 \\
f_{1}
\end{array}\right)\left[\left(\begin{array}{c}
0 \\
0 \\
f_{2}
\end{array}\right)\right]=\left(\begin{array}{c}
0 \\
0 \\
f_{1}^{\prime}
\end{array}\right)\left[\left(\begin{array}{c}
0 \\
0 \\
f_{2}^{\prime}
\end{array}\right)\right] .
$$

One finally obtains the $\mathrm{SU}(3)$ simplest-like little Higgs with $\theta \rightarrow \theta^{\prime}$ as

$$
\phi^{(1)}\left[\phi^{(2)}\right]=\mathrm{e}^{\frac{i f_{2}^{\prime}}{f_{1} f^{\prime}}\left[\frac{i f_{1}^{\prime}}{f_{2}^{\prime} f^{\prime}}\right]}\left(\begin{array}{ccc}
0 & 0 & H^{\prime} \\
0 & 0 & \\
H^{\dagger} & 0
\end{array}\right)\left[\left(\begin{array}{ccc}
0 & 0 & H \\
0 & 0 & H \\
H^{\prime \dagger} & 0
\end{array}\right) \phi_{0}^{(1)}\left[\phi_{0}^{(2)}\right],\right.
$$

where $f_{i}^{\prime}=\frac{1}{\sqrt{\pi R}} f_{i}, f^{\prime 2}=f_{1}^{\prime 2}+f_{2}^{\prime 2}, H\left(H^{\prime}\right)=\frac{1}{\sqrt{\pi R}} h\left(h^{\prime}\right)$.

\subsection{The Lower-Near-Brane SU(3) One-by-One and Collective Breaking Potentials and Higgses}

Eq. (27) can be rewritten in the following form,

$$
\begin{aligned}
& V_{y N P}^{(6)}=V_{y N P}^{(6)}(i=j)+V_{y N P}^{(6)}(i \neq j), \\
& V_{y N P}^{(6)}(i=j)=\lambda_{y N P}^{(6)}\left(\widetilde{\Phi}_{+}^{(i)^{\prime} \dagger} \widetilde{\Phi}_{+}^{(i) '}\right)^{2}, i=1,2, \\
& V_{y N P}^{(6)}(i \neq j)=\lambda_{y N P}^{(6)}\left(\widetilde{\Phi}_{+}^{(1)^{\prime} \dagger} \widetilde{\Phi}_{+}^{(2)} \widetilde{\Phi}_{+}^{(2) ' \dagger} \widetilde{\Phi}_{+}^{(1)^{\prime}}+\widetilde{\Phi}_{+}^{(2)^{\prime} \dagger} \widetilde{\Phi}_{+}^{(1)^{\prime}} \widetilde{\Phi}_{+}^{(1)^{\prime} \dagger} \widetilde{\Phi}_{+}^{(2)^{\prime}}\right),
\end{aligned}
$$

while Eq. (32) can also be expressed as follows

$$
\mathrm{V}_{\mathrm{yNP}}^{(6)}(\mathrm{i}=\mathrm{j})=\mathrm{V}_{\mathrm{yNP}(0)}^{(6)}+\mathrm{V}_{\mathrm{yNP}(1)}^{(6)}+\mathrm{V}_{\mathrm{yNP}(2)}^{(6)} \text {. }
$$

In case the term $\left([1]+i Q_{i} \alpha\right)\left(f_{i}^{\prime} / f_{j}^{\prime}\right)^{2} \theta_{i}^{\prime 2}, i \neq j=1,2$ becomes significant with respect to $\left(f_{i}^{\prime} / f_{j}^{\prime}\right) \theta_{i}^{\prime} Q_{i} \alpha, i \neq j=1,2$, then $\widetilde{\Phi}_{+}^{(1)^{\prime}}$ (with its element $\left.\phi^{(1)}\right)$ and $\widetilde{\Phi}_{+}^{(2)^{\prime}}$ (with its element $\phi^{(2)}$ ) have different rates in achieving the shift symmetry breaking, i.e. one-by-one breaking.

With $\mathrm{V}_{\mathrm{yNP}(0)}^{(6)}=\mathrm{f}_{1}^{\prime 4}+\mathrm{f}_{2}^{\prime 4} \quad$ (neglectable with correct adjustment) one can consequently treat each remaining term separately, for farther-from-brane space, after substituting Eq. (29) and (30) into the equations below,

$$
\begin{aligned}
& V_{\mathrm{yNP}(1)}^{(6)}=\lambda_{\mathrm{yNP}(\mathrm{i}=\mathrm{j}=1)}^{(6)}\left\{2\left(\phi_{0}^{(1) \dagger} \phi_{0}^{(1)}\right) \phi^{(1) \dagger} \phi^{(1)}+\left(\phi^{(1) \dagger} \phi^{(1)}\right)^{2}\right\}, \\
& V_{\mathrm{yNP}(2)}^{(6)}=\lambda_{\mathrm{yNP}(\mathrm{i}=\mathrm{j}=2)}^{(6)}\left\{2 \phi^{(2) \dagger} \phi^{(2)}\left(\phi_{0}^{(2) \dagger} \phi_{0}^{(2)}\right)+\left(\phi^{(2) \dagger} \phi^{(2)}\right)^{2}\right\},
\end{aligned}
$$


and immediately get the following potentials

$$
\begin{aligned}
\mathrm{V}_{\mathrm{yNP}(1)}^{(6)}\left[\mathrm{V}_{\mathrm{yNP}(2)}^{(6)}\right]= & \lambda_{\mathrm{yNP}}^{(6)}\left(\frac{2 \mathrm{f}_{1}^{\prime 2} \mathrm{f}_{2}^{\prime 2}}{\mathrm{f}^{2}}+\frac{\mathrm{f}_{2}^{\prime 4}\left[\mathrm{f}_{1}^{\prime 4}\right]}{4 \mathrm{f}^{4} 4}\left(\mathrm{v}^{\prime \prime}\right)^{2}\right) \mathrm{H}^{\prime \dagger} \mathrm{H}^{\prime \prime}+ \\
& \lambda_{\mathrm{yNP}}^{(6)} \frac{\mathrm{f}^{4}\left[\mathrm{f}_{1}^{4}\right]}{4 \mathrm{f}^{4} 4}\left(\mathrm{H}^{\prime \prime} \mathrm{H}^{\prime \prime}\right)^{2},
\end{aligned}
$$

where $\mathrm{H}^{\prime \prime}=\mathrm{H}^{\prime}-\mathrm{H}$, and

$$
\left\langle\mathrm{H}^{\prime \prime}\right\rangle=\frac{1}{\sqrt{2}}\left(\begin{array}{c}
0 \\
\mathrm{v}^{\prime \prime}
\end{array}\right), \quad \operatorname{Tr}\left(\mathrm{H}^{\prime \prime} \mathrm{H}^{\prime \prime}\right)=\frac{1}{2}\left(\mathrm{v}^{\prime \prime}\right)^{2},
$$

with $\mathrm{O}\left(\mathrm{v}^{\prime \prime}\right) \sim \mathrm{O}(100 \mathrm{GeV})$ and $\lambda_{\mathrm{yNP}}^{(6)} \rightarrow \lambda^{(3)}$ due to the triplets in Eqs. (29-30).

With $\mathrm{m}_{\mathrm{H}_{1}^{\prime \prime}}^{2} \sim \mathrm{m}_{\mathrm{H}_{2}^{\prime \prime}}^{2}$ and $\lambda_{y N P}{ }^{(6)}$ replaced by $\lambda^{(3)}$, it follows,

$$
\mathrm{m}_{\mathrm{H}_{\mathrm{i}}^{\prime \prime}}^{2}=\frac{\mathrm{g}^{\prime 4}}{16 \pi^{2}} \frac{\lambda^{(3)}}{\mathrm{f}^{2}}\left(2 \mathrm{f}_{1}^{\prime 2} \mathrm{f}_{2}^{\prime 2}\right) \log \left(\frac{\Lambda_{(3)}^{2}}{\mu_{\mathrm{H}_{\mathrm{i}}^{\prime \prime}}^{2}}\right), \quad \mathrm{i}=1,2,
$$

$\mathrm{O}\left(\mu_{\mathrm{H}_{\mathrm{i}}}{ }^{\prime \prime}\right) \sim \mathrm{O}\left(\mathrm{v}^{\prime \prime}\right) \sim \mathrm{O}(100 \mathrm{GeV})$. If the $\mathrm{SU}(6)$ parameters, $\Lambda_{(6)}^{Z P}, g$, and $\lambda_{\mu P}^{6}$, are replaced with $\mathrm{SU}(3)$ parameters, $\Lambda^{(3)}, g$ ' and $\lambda^{(3)}$, then Eq. (39) can be seen as a pair of SU(6)-origin light Higgses brought from $\mathrm{SU}(6)$-level [7] down to $\mathrm{SU}(3)$ level. This pairing condition is important for understanding the multicomponent Higgs that shows up as an intermediate Higgs.

Shift symmetry breaking happens for $\widetilde{\Phi}_{+}^{(1)^{\prime}}$ and $\widetilde{\Phi}_{+}^{(2)^{\prime}}$ at the same time, and contribution of its elements, $\phi^{(1)}$ and $\phi^{(2)}$, takes place simultaneously. This is the only way $V_{y N P}^{(6)}(i \neq j)$ can have stable and fixed values but, as a side effect, $V_{y N P(1)}^{(6)}$ and $V_{y N P(2)}^{(6)}$ in Eq. (34) must be included on top of $V_{y N P}^{(6)}(i \neq j)$ under a single vacuum. Excluding the constant potential $\mathrm{V}_{\mathrm{yNP}(0)}^{(6)}$, one finally has

$$
\mathrm{V}_{\mathrm{yNP}}^{(6)^{\prime}}=\mathrm{V}_{\mathrm{yNP}}^{(6)}(\mathrm{i} \neq \mathrm{j})+\mathrm{V}_{\mathrm{yNP}(1)}^{(6)}+\mathrm{V}_{\mathrm{yNP}(2)}^{(6)}
$$

where $V_{y N P}^{(6)}(i \neq j)$ can be separated into $V_{y N P(3)}^{(6)}$ and $V_{y N P(4)}^{(6)}$, in other words, $\mathrm{V}_{\mathrm{yNP}}^{(6)}(\mathrm{i} \neq \mathrm{j})$ can be rewritten in split triplets with $i \neq j$ and the factors $\phi_{0}^{(\mathrm{i})} \phi^{(\mathrm{j})}, \phi^{(\mathrm{i})} \phi_{0}^{(\mathrm{j})}$ as follows,

$$
\begin{gathered}
V_{y N P(3)}^{(6)}=V_{y N P 1(i \neq j)}^{(6)}=\lambda_{y N P}^{(6)}\left(\widetilde{\Phi}_{+}^{(1)^{\prime} \dagger} \widetilde{\Phi}^{(2)^{\prime}}\right)\left(\widetilde{\Phi}^{(2)^{\prime} \dagger} \widetilde{\Phi}_{+}^{(1)^{\prime}}\right) \\
=\lambda_{y N P}^{(6)}\left\{\left(\phi_{0}^{(1) \dagger} \phi^{(2)}\right)\left(\phi^{(2) \dagger} \phi_{0}^{(1)}\right)+\left(\phi^{(1) \dagger} \phi_{0}^{(2)}\right)\left(\phi_{0}^{(2) \dagger} \phi^{(1)}\right)+\right. \\
\left.+\left(\phi_{0}^{(1) \dagger} \phi^{(2)}\right)\left(\phi_{0}^{(2) \dagger} \phi^{(1)}\right)+\left(\phi^{(1) \dagger} \phi_{0}^{(2)}\right)\left(\phi^{(2) \dagger} \phi_{0}^{(1)}\right)\right\}
\end{gathered}
$$




$$
\begin{aligned}
V_{y N P(4)}^{(6)}= & V_{y N P 2(i \neq j)}^{(6)}=\lambda_{y N P}^{(6)}\left(\widetilde{\Phi}_{+}^{(2)^{\prime} \dagger} \widetilde{\Phi}^{(1)^{\prime}}\right)\left(\widetilde{\Phi}^{(1)^{\prime} \dagger} \widetilde{\Phi}_{+}^{(2)^{\prime}}\right) \\
= & \lambda_{\mathrm{yNP}}^{(6)}\left\{\left(\phi_{0}^{(2) \dagger} \phi^{(1)}\right)\left(\phi^{(1) \dagger} \phi_{0}^{(2)}\right)+\left(\phi^{(2) \dagger} \phi_{0}^{(1)}\right)\left(\phi_{0}^{(1) \dagger} \phi^{(2)}\right)+\right. \\
& \left.\quad+\left(\phi_{0}^{(2) \dagger} \phi^{(1)}\right)\left(\phi_{0}^{(1) \dagger} \phi^{(2)}\right)+\left(\phi^{(2) \dagger} \phi_{0}^{(1)}\right)\left(\phi^{(1) \dagger} \phi_{0}^{(2)}\right)\right\} .
\end{aligned}
$$

Adding and simplifying Eq. (41) and (42) and defining the following,

$\mathrm{V}_{\mathrm{H}(1)}^{(3)}=\lambda_{\mathrm{yNP}}^{(6)}\left\{\left(\phi_{0}^{(1) \dagger} \phi^{(2)}\right)\left(\phi^{(2) \dagger} \phi_{0}^{(1)}\right)+\left(\phi_{0}^{(2) \dagger} \phi^{(1)}\right)\left(\phi^{(1) \dagger} \phi_{0}^{(2)}\right)\right\}$

$\mathrm{V}_{\mathrm{H}(2)}^{(3)}=\lambda_{\mathrm{yNP}}^{(6)}\left\{\left(\phi^{(1) \dagger} \phi_{0}^{(2)}\right)\left(\phi_{0}^{(2) \dagger} \phi^{(1)}\right)+\left(\phi^{(2) \dagger} \phi_{0}^{(1)}\right)\left(\phi_{0}^{(1) \dagger} \phi^{(2)}\right)\right\}$,

gives the mass terms and constant potentials, after adjusting $\lambda_{\mathrm{yNP}}^{(6)} \rightarrow \lambda^{(3)}$,

$$
\mathrm{V}_{\mathrm{H}}^{(3)}=\lambda^{(3)}\left\{4 \mathrm{f}_{1}^{\prime 2} \mathrm{f}_{2}^{\prime 2}+\frac{\mathrm{f}_{1}^{\prime 4}+\mathrm{f}_{2}^{\prime 4}}{2 \mathrm{f}^{\prime 2}} \mathrm{v}^{\prime \prime 2}+\frac{\mathrm{f}_{1}^{\prime 4}+\mathrm{f}_{2}^{4}}{\mathrm{f}^{\prime 2}} \mathrm{H}^{\prime \prime \dagger} \mathrm{H}^{\prime \prime}\right\},
$$

where $\mathrm{O}\left(\mathrm{V}^{\prime \prime}\right) \sim \mathrm{O}(100 \mathrm{GeV})$ and $\mathrm{V}_{\mathrm{H}^{\prime \prime}}^{(3)}=\mathrm{V}_{\mathrm{H}(1)}^{(3)}+\mathrm{V}_{\mathrm{H}(2)}^{(3)}$. The remaining terms of Eq. (41) and (42) give, respectively,

$\mathrm{V}_{\mathrm{C}(1)}^{(3)}=\lambda_{\mathrm{yNP}}^{(6)}\left\{\left(\phi_{0}^{(1) \dagger} \phi^{(2)}\right)\left(\phi_{0}^{(2) \dagger} \phi^{(1)}\right)+\left(\phi_{0}^{(2) \dagger} \phi^{(1)}\right)\left(\phi_{0}^{(1) \dagger} \phi^{(2)}\right)\right\}$
$\mathrm{V}_{\mathrm{C}(2)}^{(3)}=\lambda_{\mathrm{yNP}}^{(6)}\left\{\left(\phi^{(1) \dagger} \phi_{0}^{(2)}\right)\left(\phi^{(2) \dagger} \phi_{0}^{(1)}\right)+\left(\phi^{(2) \dagger} \phi_{0}^{(1)}\right)\left(\phi^{(1) \dagger} \phi_{0}^{(2)}\right)\right\}$

which can be expressed, setting $\Delta f^{\prime}=f_{2}^{\prime}-f_{1}^{\prime},\left(f_{2}^{\prime}>f_{1}^{\prime}\right), \frac{f_{2}^{\prime}}{f_{1}^{\prime}}\left[\frac{f_{1}^{\prime}}{f_{2}^{\prime}}\right]=1+$ $\frac{1}{f_{1}^{\prime}}\left[-\frac{1}{f_{2}^{\prime}}\right] \Delta f^{\prime}$ and $\lambda_{y N P}^{(6)} \rightarrow \lambda^{(3)}$, as follows,

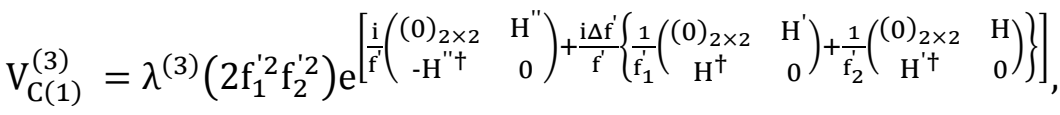

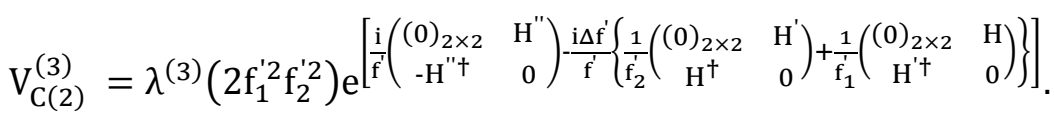

Eq. (48) and (49) immediately give the following potential,

$$
\mathrm{V}_{\mathrm{C}}^{(3)}=\mathrm{V}_{\mathrm{C}(1)}^{(3)}+\mathrm{V}_{\mathrm{C}(2)}^{(3)} \text {, }
$$

and the total potential becomes

$$
\mathrm{V}_{\mathrm{yNP}(\mathrm{i} \neq \mathrm{j})}^{(6)}=\mathrm{V}_{\mathrm{yNP}(3)}^{(6)}+\mathrm{V}_{\mathrm{yNP}(4)}^{(6)}=\mathrm{V}_{\mathrm{H}^{\prime \prime}}^{(3)}+\mathrm{V}_{\mathrm{C}}^{(3)} .
$$

For indentical $H^{\prime}$ and $H$ one can set $\mathrm{H}^{\prime} \sim \mathrm{H}$ and $H^{\prime \prime}=H^{\prime}-\mathrm{H} \sim 0$, which causes one $\mathrm{SU}(3)$ symmetry to be conserved, as shown by $V_{C}^{(3)} \sim 0$. 
Theoretically $H$ (or $H^{\prime}$ ) can achieve non-zero mass as shown by Eq. (48) and (49), which are written in short form as given below,

$$
\mathrm{V}_{\mathrm{C}(1)}^{(3)}\left[\mathrm{V}_{\mathrm{C}(2)}^{(3)}\right]=\left(2 \mathrm{f}_{1}^{\prime 2} \mathrm{f}_{2}^{\prime 2}\right) \exp \left[-\frac{\mathrm{i}}{\mathrm{f}^{\prime}}\left[\frac{\mathrm{i}}{\mathrm{f}^{\prime}}\right] \frac{\mathrm{f}_{1}^{\prime 2}-\mathrm{f}_{2}^{\prime 2}}{\mathrm{f}_{1}^{\prime 2} \mathrm{f}_{2}^{\prime 2}}\left(\begin{array}{cc}
(0)_{2 \times 2} & \mathrm{H} \\
\mathrm{H}^{\dagger} & 0
\end{array}\right)\right] \text {, }
$$

and can be expanded in $H\left[H^{\prime}\right]$ to produce the mass term $H^{\dagger} H\left[H^{\prime \dagger} H^{\prime}\right]$. From the second and fourth order terms of $V_{C}^{(3)}$ defined in Eq. (50) one can rewrite it, after making use of Eq. (52), to give

$$
V_{C}^{(3)}=\lambda^{(3)}\left\{-\frac{2\left(f_{1}^{\prime 2}-f_{2}^{\prime 2}\right)^{2}}{f^{\prime 2}}+\frac{v^{\prime \prime \prime 2}\left(f_{1}^{\prime}-f_{2}^{\prime}\right)^{4}}{6 f^{4} f_{1}^{\prime 2} f_{2}^{\prime 2}}\right\} H^{\dagger} H+\lambda^{(3)} \frac{\left(f_{1}^{\prime 2}-f_{2}^{\prime 2}\right)^{4}}{6 f_{1}^{\prime 2} f_{2}^{\prime 2} f^{4} 4}\left(H^{\dagger} H\right)^{2},
$$

where $\left[v^{\prime \prime 2}\left(\mathrm{f}_{1}^{\prime 2}-\mathrm{f}_{2}^{\prime 2}\right)^{2}\right] /\left[12 \mathrm{f}^{\prime 2} \mathrm{f}_{1}^{\prime 2} \mathrm{f}_{2}^{\prime 2}\right] \ll 1,\langle\mathrm{H}\rangle=\frac{1}{\sqrt{2}}\left(\begin{array}{c}0 \\ \mathrm{v}^{\prime \prime \prime}\end{array}\right)$, and

$$
\operatorname{Tr}\left\langle\mathrm{HH}^{\dagger}\right\rangle=\frac{1}{2}\left(\mathrm{v}^{\prime \prime \prime}\right)^{2}, \mathrm{O}\left(\mathrm{v}^{\prime \prime \prime}\right) \sim 0(100 \mathrm{GeV}) \text {. }
$$

The mass term (quartic term) in $H^{\dagger} H\left[\left(H^{\prime \dagger} H^{\prime}\right)^{2}\right]$ vanishes only if $f_{1}{ }^{\prime}=f_{2}$ ' or becomes negligible if $f_{1}^{\prime} \sim f_{2}$. The conclusion can be drawn from now on that the only way the potential $V_{C}^{(3)}$ does not become zero is by setting $f_{1}^{\prime} \neq f_{2}$ ', which provides a negative mass term of spontaneous symmetry breaking (SSB) potential. This indicates the existence of a new global symmetry $U(1)_{\mathrm{VH}}$, the VEV homogeneity symmetry, which connects with $V_{C}^{(3)}=0$ and is broken when $f_{1}^{\prime} \neq f_{2}{ }^{\prime}$. This will play an important role in the restoration of one $\mathrm{SU}(3)$ to provide the final global symmetry $\mathrm{SU}(6) \rightarrow \mathrm{SU}(3) \times \mathrm{SU}(3) \times \mathrm{U}(1) \rightarrow \mathrm{SU}(3) \times$ $\mathrm{SU}(2) \times \mathrm{U}(1)_{\mathrm{VH}}$, which strongly shows the breaking of the single $\mathrm{SU}(3)$ vacuum. Collective breaking with the single vacuum requires VEV homogeneity $f_{1}$ ' $\sim f_{2}$ ' or $V_{C}^{(3)} \sim 0$, which makes the collective potential in Eq. (40) become

$$
\mathrm{V}_{\mathrm{yNP}}^{(6)^{\prime}}=\mathrm{V}_{\mathrm{H}^{\prime \prime}}^{(3)}+\mathrm{V}_{\mathrm{yNP}(1)}^{(6)}+\mathrm{V}_{\mathrm{yNP}(2)}^{(6)}
$$

with $\mathrm{V}_{\mathrm{H}}^{(3)}$ and $\mathrm{V}_{\mathrm{yNP}(\mathrm{i})}^{(6)}, \mathrm{i}=1,2$, as shown by Eq. (45) and (37) respectively.

Therefore Eq. (54) straightforwardly provides the following potential,

$$
\begin{aligned}
V_{H^{\prime \prime}}^{(3)}= & \lambda^{(3)}\left(\frac{4 f_{1}^{\prime 2} f_{2}^{\prime 2}}{f^{\prime 2}}+\frac{f_{1}^{\prime 4}+f_{2}^{\prime 4}}{4 f^{\prime 4}} v^{\prime \prime 2}+\frac{f_{1}^{\prime 4}+f_{2}^{\prime 4}}{f^{\prime 2}}\right) H^{\prime \prime \dagger} H^{\prime \prime} \\
& +\lambda^{(3)} \frac{f_{1}^{\prime 4}+f_{2}^{\prime 4}}{4 f^{4} 4}\left(H^{\prime \prime} H^{\prime \prime}\right)^{2},
\end{aligned}
$$

and the Higgs mass-squared,

$$
\mathrm{m}_{\mathrm{H}^{\prime \prime}}^{2}=\frac{\mathrm{g}^{\prime 4}}{16 \pi^{2}} \frac{\lambda^{(3)}}{\mathrm{f}^{\prime 2}}\left\{4 \mathrm{f}_{1}^{\prime 2} \mathrm{f}_{2}^{\prime 2}+\left(\mathrm{f}_{1}^{\prime 4}+\mathrm{f}_{2}^{\prime 4}\right)\right\} \log \left(\frac{\Lambda_{(3)}^{2}}{\mu_{\mathrm{H}^{\prime \prime}}^{2}}\right) .
$$


Considering $\Lambda^{(3)} \ll \Lambda_{(6)}^{Z P}, 4 f_{1}^{\prime 2} f_{2}^{\prime 2}$ in addition to $\left(f_{1}^{\prime}{ }^{4}+f_{2}^{\prime}\right)$, where $f_{1}^{\prime} f_{2}^{\prime 2}$ and $f_{1}^{\prime}{ }^{4}+f_{2}^{\prime}{ }_{2}^{4}$ are dominant factors in the light Higgs and the intermediate Higgs mass-squared [7], one concludes that Eq. (56) provides the mass of the intermediate Higgs boson from the $\mathrm{SU}(3)_{\mathrm{CH}} \times \mathrm{SU}(3)_{\mathrm{PH}}$ symmetry that emerges under the collective breaking property. Comparing the one-by-one breaking pattern in the closer-to-brane and the farther-from-brane parts of the lower-nearbrane, one immediately notices that a collective breaking (simultaneous breaking) is just a special condition of the one-by-one breaking of the shift symmetry with the time difference being very short. In this way one can regard Eq. (56) not only as a single Higgs but rather as a multi-component Higgs that consists of two scalar-pairs and one extra scalar.

\section{SU(6) Strongly-Coupled-Origin NGB-Dual Higgs}

\subsection{Higgs-NGB Double Vacua as a Source of NGB-Dual Higgs}

The AdS/CFT correspondence suggests the correspondence of 5D $(y \sim 0)$ local gauge and 4D global gauge $\mathrm{SU}(6)$ symmetry [1], which in this context extends to a 4D PNB Higgs and a 5D ( $y \sim 0)$ Nambu-Goldstone boson (NGB) due to global and local properties. Consequently one can generalize and interpret $\widetilde{\Phi}^{(j)}, \mathrm{j}=1,2$ in Eq. (20) to include NGB, $\xi$, which can be put in a diagonal matrix representation, taking into consideration the PNBs residing in the offdiagonal of the sub-matrices, as shown by Eq. (9).

One can use $\mathrm{SU}(6)$ generators $\lambda_{8}, \lambda_{34}, \lambda_{35}$ to define NGBs as follows,

$$
\xi^{(1)}=\xi\left(n_{8} \lambda_{8}+n_{35} \lambda_{35}\right), \quad \xi^{(2)}=\xi\left(n_{34} \lambda_{34}+n_{35} \lambda_{35}\right),
$$

where $\xi$ is the NGB, $n_{8}, n_{34}$ and $n_{35}$ are normalization constants, and the generators are given below [21],

$$
\begin{gathered}
\lambda_{8}=\frac{1}{\sqrt{3}}\left(\begin{array}{ll}
\operatorname{diag}(1,1,-2) & (0)_{3 \times 3} \\
(0)_{3 \times 3} & (0)_{3 \times 3}
\end{array}\right), \quad \lambda_{34}=\frac{1}{\sqrt{3}}\left(\begin{array}{l}
(0)_{3 \times 3}(0)_{3 \times 3} \\
(0)_{3 \times 3} \operatorname{diag}(1,1,-2)
\end{array}\right), \\
\lambda_{35}=\frac{1}{\sqrt{3}}\left(\begin{array}{ll}
\operatorname{diag}(-1,-1,-1) & (0)_{3 \times 3} \\
(0)_{3 \times 3} & \operatorname{diag}(1,1,1)
\end{array}\right)
\end{gathered}
$$

so that,

$$
\xi^{(1)}=\xi \operatorname{diag}(3,3,0,-2,-2,-2), \xi^{(2)}=\xi \operatorname{diag}(-2,-2,-2,3,3,0) .
$$

Defining $\xi^{\prime}=3 \xi, \xi_{0}=-2 \xi, \quad\left(\xi^{\prime}\right)_{n g}=\operatorname{diag}\left(\xi^{\prime}, \xi^{\prime}, 0\right)$, and $\xi_{0}^{\prime}=\xi_{0} I_{3 \times 3}, \operatorname{SU}(6)$ NGBs can be written in short form as, 


$$
\xi^{(1)}=\left(\begin{array}{ll}
\left(\left(\xi^{\prime}\right)_{\mathrm{ng}}\right)_{3 \times 3} & (0)_{3 \times 3} \\
(0)_{3 \times 3} & \left(\left(\xi^{\prime}\right)_{0}\right)_{3 \times 3}
\end{array}\right), \quad \xi^{(2)}=\left(\begin{array}{ll}
\left(\left(\xi^{\prime}\right)_{0}\right)_{3 \times 3} & (0)_{3 \times 3} \\
(0)_{3 \times 3} & \left(\left(\xi^{\prime}\right)_{\mathrm{ng}}\right)_{3 \times 3}
\end{array}\right)
$$

Recalling Eq. (16) with $i \neq j$, the Coleman-Weinberg potential is generalized by replacing $\widetilde{\Phi}^{(j)}, j=1,2$ with $\Phi_{N G}^{(j)}, j=1,2$, which is justified considering $\Phi_{N G}^{(j)}, j=1,2$ is the third component of a massive 4D gauge boson, while $\widetilde{\Phi}^{(i)}, i=1,2$ is the PNB Higgs.

Substitution of the fifth component of the 5D gauge boson with the third component is definitely acceptable [22], which gives a generalized ColemanWeinberg potential for $\mathrm{SU}(6) \widetilde{\Phi}^{(i)}, i=1,2$ as below,

$$
\mathrm{V}_{\mathrm{yg}}^{(6)}=\lambda_{\mathrm{y}}^{(6)}\left(\widetilde{\Phi}^{(\mathrm{i}) \dagger} \Phi_{\mathrm{NG}}^{(\mathrm{j})}\right)\left(\Phi_{\mathrm{NG}}^{(\mathrm{j}) \dagger} \widetilde{\Phi}^{(\mathrm{i})}\right), \quad \mathrm{i}, \mathrm{j}=1,2,
$$

and for $\operatorname{SU}(6) \widetilde{\Phi}^{(i) \prime}, i=1,2$,

$$
V_{N P g}^{(6)}=\lambda_{y N P}^{(6)}\left(\widetilde{\Phi}^{(i)^{\prime} \dagger} \Phi_{N G}^{(j)}\right)\left(\Phi_{N G}^{(j) \dagger} \widetilde{\Phi}^{(i)^{\prime}}\right), \quad i, j=1,2,
$$

where $\Phi_{N G}^{(j)}$ is written as,

$$
\Phi_{N G}^{(j)}=v_{N G}^{(j)} e^{\frac{i f_{i}^{\prime}}{f_{j}^{\prime}} f^{(j)}}, i \neq j=1,2
$$

with + for $i=1,-$ for $i=2, v_{N G}^{(1)}=v$, and $v_{N G}^{(2)}=v^{\prime}$ in Eq. (7), or $\mathrm{v}_{\mathrm{NG}}^{(1)}=$ $\left(\begin{array}{llllll}0 & 0 & f_{1}^{\prime} & 0 & 0 & 0\end{array}\right)^{\mathrm{T}}$ and $\mathrm{v}_{\mathrm{NG}}^{(2)}=\left(\begin{array}{llllll}0 & 0 & 0 & 0 & 0 & \mathrm{f}_{2}^{\prime}\end{array}\right)^{\mathrm{T}}$. Applying a similar expansion to Eq. (10) to obtain Eq. (28), one gets

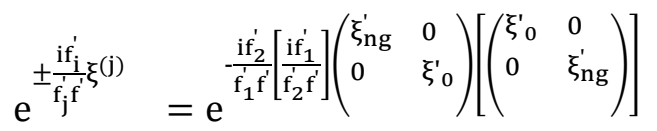

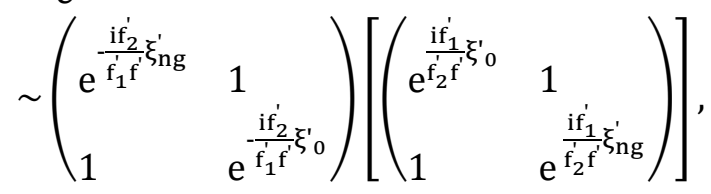

which gives directly, with the aid of Eq. (7), the following sextets,

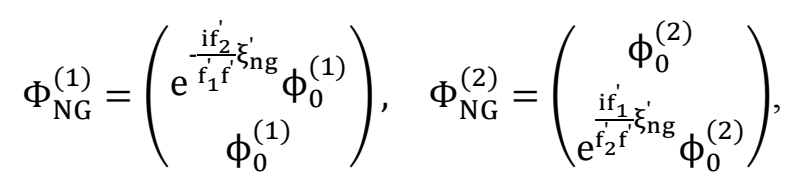

where one can define $\mathrm{SU}(3) \operatorname{NGBs} \phi_{N G}^{(j)}, j=1,2$ as follows,

$$
\phi_{\mathrm{NG}}^{(1)}\left[\phi_{\mathrm{NG}}^{(2)}\right]=\mathrm{e}^{-\frac{i \mathrm{f}_{2}^{\prime}}{\mathrm{f}_{1} \mathrm{f}}\left[\frac{i \mathrm{f}_{1}^{\prime}}{\mathrm{f}_{2}}\right]} \dot{\xi}_{\mathrm{ng}}^{\prime} \phi_{0}^{(1)}\left[\phi_{0}^{(2)}\right]
$$


Absorbing factor $1 / \sqrt{2}$ by $\left(\xi_{n g}^{\prime}\right)_{j}=\left(\frac{1}{\sqrt{2}} \xi^{\prime}\right), j=1,2$, Eq. (66) resembles the NGBs produced in $\mathrm{SU}(3) \times \mathrm{SU}(3)$ symmetry as discussed in [22].

In fact, Eq. (65) can be rewritten into a compact form similar to Eq. (28),

$$
\Phi_{\mathrm{NG}}^{(1)}=\left(\begin{array}{c}
\phi_{\mathrm{NG}}^{(1)} \\
\phi_{0}^{(1)}
\end{array}\right), \quad \Phi_{\mathrm{NG}}^{(2)}=\left(\begin{array}{c}
\phi_{0}^{(2)} \\
\phi_{\mathrm{NG}}^{(2)}
\end{array}\right) .
$$

In comparison, one finds that the doublets in Eq. (67) are the inversion of the doublets in Eq. (28) after replacing $\phi^{(1)}{ }_{N G}$ with $\phi^{(1)}$, indicating 2 different vacua with 2-VEV, where one belongs to the PNB Higgs and the other belongs to the NGB field.

Summing up Eq. (28) and (67) gives

$$
\widetilde{\Phi}^{(1)^{\prime}}+\Phi_{\mathrm{NG}}^{(1)}=\left(\begin{array}{c}
\phi_{0}^{(1)}+\phi_{\mathrm{NG}}^{(1)} \\
\phi_{0}^{(1)}+\phi^{(1)}
\end{array}\right), \quad \widetilde{\Phi}^{(2)^{\prime}}+\Phi_{\mathrm{NG}}^{(2)}=\left(\begin{array}{c}
\phi_{0}^{(2)}+\phi^{(2)} \\
\phi_{0}^{(2)}+\phi_{\mathrm{NG}}^{(2)}
\end{array}\right),
$$

where each VEV $\phi^{(\mathrm{i})}, i=1,2$ is shifted by both Higgs and NGB indicating the existence of a double vacua with a 2-VEV system [22-23].

\subsection{NGB-Dual Higgses Masses from Triplet-Triplet Splitting}

The triplet-triplet splitting potential is obtained with the aid of Eq. (27) and (62) and it indicates the emerging of a massive particle which can be either a PNB Higgs or an NGB-dual Higgs.

Let's start by rewriting the potential as, $(i, j=1,2)$,

$$
\begin{aligned}
& V_{N P g}^{(6)}=V_{N P g(i=j)}^{(6)}+V_{N P g(i \neq j)}^{(6)}, \\
& V_{N P g(i=j)}^{(6)}=\lambda_{y N P}^{(6)}\left(\widetilde{\Phi}^{(i) \dagger} \Phi_{N G}^{(i)} \Phi_{N G}^{(i) \dagger} \widetilde{\Phi}^{(i)^{\prime}}+\Phi_{N G}^{(i) \dagger} \widetilde{\Phi}^{(i)^{\prime}} \widetilde{\Phi}^{(i)^{\prime} \dagger} \Phi_{N G}^{(i)}\right), \\
& V_{N P g(i \neq j)}^{(6)}=\lambda_{y N P}^{(6)}\left(\widetilde{\Phi}^{(i) \dagger} \Phi_{N G}^{(j)} \Phi_{N G}^{(j) \dagger} \widetilde{\Phi}^{(i)^{\prime}}+\Phi_{N G}^{(j) \dagger} \widetilde{\Phi}^{(i)^{\prime}} \widetilde{\Phi}^{(i)^{\prime} \dagger} \Phi_{N G}^{(j)}\right) .
\end{aligned}
$$

Substituting Eq. (28) and (67) into Eq. (70), neglecting mixed terms such as $\phi_{0}^{(i) \dagger} \phi_{N G}^{(i)} \phi_{0}^{(i) \dagger} \phi^{(i)}, \quad \phi^{(i) \dagger} \phi_{0}^{(i)} \phi_{N G}^{(i) \dagger} \phi_{0}^{(i)}, \quad \phi_{N G}^{(i) \dagger} \phi_{0}^{(i)} \phi^{(i) \dagger} \phi_{0}^{(i)}, \quad$ and $\phi_{0}^{(i) \dagger} \phi^{(i)} \phi_{0}^{(i) \dagger} \phi_{N G}^{(i)}$ (this produces the terms $\left(\theta^{\prime}+\xi_{\mathrm{ng}}^{\prime}\right),\left(\theta^{\prime \dagger}+\xi_{\mathrm{ng}}^{\prime}\right)$ and their linear combinations, which facilitate double vacua with double shifts as shown by Eq. (68) and can be regarded as NGB shift symmetry [23]), one can simplify Eq. (70), replacing $\lambda_{y N P}^{(6)}$ with $\lambda^{(3)}$, as 


$$
\begin{aligned}
V_{N P g(i=j)}^{(6)}= & \lambda^{(3)}\left\{\left(\phi_{0}^{(i) \dagger} \phi_{N G}^{(i)} \phi_{N G}^{(i) \dagger} \phi_{0}^{(i)}+\phi_{N G}^{(i) \dagger} \phi_{0}^{(i)} \phi_{0}^{(i) \dagger} \phi_{N G}^{(i)}\right)+\right. \\
& \left.\left(\phi^{(i) \dagger} \phi_{0}^{(i)} \phi_{0}^{(i) \dagger} \phi^{(i)}+\phi_{0}^{(i) \dagger} \phi^{(i)} \phi^{(i) \dagger} \phi_{0}^{(i)}\right)\right\} .
\end{aligned}
$$

The first term gives the constant, the second term the mass term, as follows,

$$
\mathrm{V}_{\mathrm{NPg}(\mathrm{i}=\mathrm{j})}^{(6)}=2\left(\mathrm{f}_{1}^{\prime 4}+\mathrm{f}_{2}^{\prime 4}\right)+\frac{2 \mathrm{f}_{1}^{\prime 2} \mathrm{f}_{2}^{2}}{\mathrm{f}^{\prime 2}} \mathrm{H}^{\prime \prime} \mathrm{H}^{\prime \prime},
$$

where the mass term $H^{\prime \prime} H^{\prime \prime}$ has no influence from NGB as shown by the noNGB term in the second term. One names $H^{\prime \prime}{ }_{n g .1(i)}, i=1,2$ as the first NGBdual Higgses with the following mass squared,

$$
\mathrm{m}_{\mathrm{H}_{\text {ng.1(i) }}^{\prime \prime}}^{2}=\frac{\mathrm{g}^{\prime 4}}{16 \pi^{2}} \frac{\lambda^{(3)}}{\mathrm{f}^{2}}\left(2 \mathrm{f}_{1}^{\prime 2} \mathrm{f}_{2}^{\prime 2}\right) \log \left(\frac{\Lambda_{(3)}^{2}}{\mu_{\mathrm{H}_{\text {ng.1(i) }}^{2}}^{\prime \prime}}\right),
$$

where $\mathcal{O}\left(\mu_{H^{\prime \prime}}{ }_{n g .1(i)}\right) \sim \mathcal{O}(100 \mathrm{GeV}), g^{\prime}$ is the $\mathrm{SU}(3)$ coupling constant and $\Lambda_{(3)}$ is the cut-off scale.

On the other hand, one can also substitute Eq. (28) and (67) into Eq. (71), (neglecting mixed terms such as $\phi_{0}^{(i) \dagger} \phi_{0}^{(j)} \phi_{N G}^{(j) \dagger} \phi^{(i)}, \phi^{(i) \dagger} \phi_{N G}^{(j)} \phi_{0}^{(j) \dagger} \phi_{0}^{(i)}$, $\phi_{0}^{(j) \dagger} \phi_{0}^{(i)} \phi^{(i) \dagger} \phi_{N G}^{(j)}$, and $\phi_{N G}^{(j) \dagger} \phi^{(i)} \phi_{0}^{(i) \dagger} \phi_{0}^{(j)}$, based on the same reason as above), replacing $\lambda_{y N P}^{(6)}$ with $\lambda^{(3)}$, to get

$$
\begin{aligned}
V_{N P g(i \neq j)}^{(6)}= & \lambda^{(3)}\left\{\left(\phi_{0}^{(i) \dagger} \phi_{0}^{(j)} \phi_{0}^{(j) \dagger} \phi_{0}^{(i)}+\phi_{0}^{(j) \dagger} \phi_{0}^{(i)} \phi_{0}^{(i) \dagger} \phi_{0}^{(j)}\right)+\right. \\
& \left.\left(\phi^{(i) \dagger} \phi_{N G}^{(j)} \phi_{N G}^{(j) \dagger} \phi^{(i)}+\phi_{N G}^{(j) \dagger} \phi^{(i)} \phi^{(i) \dagger} \phi_{N G}^{(j)}\right)\right\} .
\end{aligned}
$$

The first term is a constant, while the second is the anticipated mass term as shown below,

$$
V_{N P g(i \neq j)}^{(6)}=4\left(f_{1}^{\prime 2} f_{2}^{\prime 2}\right)+\frac{f_{1}^{\prime 4}+f_{2}^{\prime 4}}{f^{\prime 2}} H^{\prime \prime \dagger} H^{\prime \prime},
$$

where one finds that the mass term $H^{\prime \prime \dagger} H^{\prime \prime}$ in Eq. (76) is obtained from the combination of Higgs and NGBs as shown by the second term in Eq. (75), which is actually the second NGB-dual Higgs, $H^{\prime \prime}{ }_{n g .2}$, having the following mass squared,

$$
m_{H_{n g .2}^{\prime \prime}}^{2}=\frac{g^{4}}{16 \pi^{2}} \frac{\lambda^{(3)}}{f^{\prime 2}}\left(f_{1}^{\prime 4}+f_{2}^{\prime 4}\right) \log \left(\frac{\Lambda_{(3)}^{2}}{\mu_{H_{n g .2}^{\prime \prime}}^{2}}\right),
$$

where $\mathcal{O}\left(\mu_{H^{\prime}{ }^{\prime}{ }_{\text {g. }}}\right) \sim \mathcal{O}(100 \mathrm{GeV})$. 
Both the PNB Higgs and the NGB-dual Higgses become the basic constituents of exotic scalars. The formation of the NGB-dual Higgses from a Higgs and NGBs, including the mass generation, requires a new mechanism, which will be discussed in a separate paper.

Before closing this subsection, note that Eq. (77) shows a stand-alone free NGB-dual Higgs, which can be regarded as an exotic single scalar that can also serve as candidate for a relic dark matter [24]. Nevertheless, one also faces the hidden NGB-dual Higgs, which can be found as a bounded scalar, joined with either a PNB Higgs or itself, to form exotic multi-component Higgses and scalars. This will also be discussed in another paper [22].

Based on Eq. (69), adding up the mass squareds in Eq. (74) and Eq. (77) with multiplying factors 2 (two) and 1 (one) respectively, one immediately retrieves Eq. (56), the mass squared of the exotic multi-component Higgs, which is now shown as a 3-scalar Higgs.

\section{Duality of NGB-Dual and PNB Higgses in a Double Vacua}

One can rewrite the sextet VEV in Eq. (7), similar to the linear sigma model, by adding the shifts in the upper (lower) triplet and non-zero VEV in the lower (upper) triplet as follows,

$$
\begin{aligned}
\left(\begin{array}{llllll}
0 & 0 & f_{1}^{\prime} & 0 & 0 & 0
\end{array}\right)^{\mathrm{T}} \rightarrow\left(\begin{array}{lllllll}
0 & 0 & f_{1}^{\prime}+\eta_{1} & \xi_{0} & \xi_{0} & \xi_{0}
\end{array}\right)^{\mathrm{T}} \\
\left(\begin{array}{llllllll}
0 & 0 & 0 & 0 & 0 & f_{2}^{\prime}
\end{array}\right)^{\mathrm{T}} \rightarrow\left(\begin{array}{lllllll}
\xi^{\prime} & \xi^{\prime} & \xi^{\prime} & 0 & 0 & f_{2}^{\prime}+\eta_{2}
\end{array}\right)^{\mathrm{T}}
\end{aligned}
$$

which yield NGBs $\xi_{0}$ and $\xi^{\prime}$ and Higgses $\eta_{1}$ and $\eta_{2}$. After triplet-triplet splitting the sextets in Eq. (78) become 2 (two) sets of triplets, as follows,

$$
\left(\begin{array}{c}
0 \\
0 \\
f_{1}^{\prime}+\eta_{1}
\end{array}\right), \xi^{0}\left(\begin{array}{l}
1 \\
1 \\
1
\end{array}\right) \text { and }\left(\begin{array}{c}
0 \\
0 \\
f_{2}^{\prime}+\eta_{2}
\end{array}\right), \xi^{\prime}\left(\begin{array}{l}
1 \\
1 \\
1
\end{array}\right)
$$

Eq. (79) reflects the main property of a double vacua, where $f_{i}^{\prime}, i=1,2$, in the first vacuum, develop a non-zero value and experience the shifts $\eta_{i}$, which become strongly-coupled simplest little-like Higgs $\phi^{(i)}$. On the other hand, the second vacuum remains in zero VEV but suffers from the shifts $\xi^{0}$ and $\xi^{\prime}$, which become NGBs, $\phi^{(j)}{ }_{N G}, j=1,2$.

Recalling the potential in Eq. (31) and Eq. (54), with $\phi^{(i)}, \phi^{(j)}, i, j=1,2$, which reflects a single-vacuum Coleman-Weinberg potential, one obtains the stronglycoupled heavy multi-component PNB Higgs in Eq. (56). On the other hand, Eq. (72) and Eq. (75) with $\phi^{(i)}, \phi^{(j)}{ }_{N G}$ or $\phi^{(i)}{ }_{0}, \phi^{(i)}{ }_{N G}$, which reflect a double vacua, produce the components of the multi-component Higgs, the NGB-dual Higgses, 
via a generalized Coleman-Weinberg potential. Eq. (79) exactly resembles Eq. (68) if the following correspondences are established,

$$
\begin{aligned}
& \left(\phi_{0}^{(\mathrm{i})}+\phi^{(\mathrm{i})}\right) \leftrightarrow\left(\begin{array}{c}
0 \\
0 \\
f_{\mathrm{i}}^{\prime}+\eta_{\mathrm{i}}
\end{array}\right), \quad \mathrm{i}=1,2, \\
& \phi_{\mathrm{NG}}^{(\mathrm{j})} \longrightarrow\left(\phi_{0}^{(\mathrm{j})}+\phi_{\mathrm{NG}}^{(\mathrm{j})}\right) \leftrightarrow \xi^{0}\left[\xi^{\prime}\right]\left(\begin{array}{l}
1 \\
1 \\
1
\end{array}\right), \mathrm{j}=1,2,
\end{aligned}
$$

where NGB shift symmetry has been utilised in Eq. (81).

In the context of a double vacua one immediately finds the duality between strongly-coupled heavy PNB Higgs, $H$ ", in Eq. (56) and 3 (three) NGB-dual Higgses, $2 H^{\prime \prime}{ }_{n g .1}$ and $1 H^{\prime \prime}{ }_{n g .2}$, in Eq. (74) and Eq. (77), which exactly resemble Eq. (56). The second behaves like the components of the first, which happens if the shifts take place in the second vacuum with zero VEV. On the other hand, the first happens if the shifts take place at non-zero VEVs in the first vacuum. Just like wave-particle duality, Nature will decide which type of Higgses will emerge, whether from a double-vacua or a single vacuum.

To conclude, the duality of Higgses is the consequence of vacuum configuration, either as single vacuum or double vacua, where each vacuum has equal probability to develop shifts with respect to zero (non-zero)-VEV vacuum.

\section{Conclusion}

Global symmetry $\mathrm{SU}(3)_{\mathrm{CH}} \times \mathrm{SU}(3)_{\mathrm{PH}} \times \mathrm{U}(1)$ as the result of $\mathrm{SU}(6)$ symmetry breaking by the will-be simplest little Higgs scalar opens further breakings in at least two patterns, i.e. one-by-one and collective breakings. The first produces two massive scalar-pair Higgses, while the second one produces a large-mass Higgs. In comparison with the previously mentioned result and also with Higgses derived from the SU(6) baby Higgs, it can be concluded that the collectively-broken Higgs is actually a 3-scalar.

A similar result can be obtained alternatively via a generalized ColemanWeinberg potential, where NGB-like scalars replace the two will-be simplest little Higgs scalars in a non-quadratic-based quartic potential. The only difference is that now the mass squareds emerge as the components of the mass squared of the strongly-coupled 3-scalar Higgs. Here, two types of NGB-dual Higgses are found. 
This phenomenon brings us directly to a double-vacua system, where the first vacuum yields Higgs fields and the second vacuum yields NGB fields. As a consequence the duality between the two types of Higgses emerges, i.e. a 3scalar Higgs from a single vacuum and 3 (three) NGB-dual Higgses as the shifts with respect to non-zero and zero VEVs in the first and second vacuum respectively of the double vacua.

Further research must be performed to analytically ascertain the above conclusion, including a verification of the Higgs wavefunction.

\section{Acknowledgements}

JSK, AH, FPZ, would like to thank ITB and ICTMP for the oppurtunity and support. JSK and FPZ also would like to thank ITB and Kemdikbud, Republic of Indonesia for their financial support, fiscal year 2014.

\section{References}

[1] Lim, C.S. \& Maru, N., Towards a Realistic Grand Gauge-Higgs Unification, Phys. Lett., B653, pp. 320-324, 2007.

[2] Burdman, G. \& Nomura, Y., Holographic Theories of Electroweak Symmetry Breaking without A Higgs Boson, Phys. Rev. D69, pp. 115013, 2004.

[3] Quiros, M., New Ideas in Symmetry Breaking, TASI 2004, Physics in D > 4, World Scientific Publishing Co., Singapore, 2006.

[4] Burdman, G. \& Nomura, Y., Unification of Higgs and Gauge Fields in Five Dimensions, Nucl. Phys., B656, pp. 3-22, 2003.

[5] Cheng, H.C., Matchev, K.T. \& Schmaltz, M., Bosonic Supersymmetry? Getting Fooled at The LHC, Phys. Rev., D66, pp. 036005, 2002.

[6] Hall, L.J., Murayama, H. \& Nomura, Y., Wilson Lines and Symmetry Breaking on Orbifolds, Nucl. Phys., B645, pp. 85-104, 2002.

[7] Hartanto, A., Zen, F.P., Kosasih, J.S. \& Handoko, L.T., Near-Brane SU(6)origin Higgs in Scherk-Schwarz Breaking of 5-Dimensional SU(6) GUT, Int. J. Mod. Phys., A27, pp. SO217751X12500352, 2012.

[8] Hosotani, Y., Dynamical Mass Generation by Compact Extra Dimensions, Phys. Lett., B126, 309-313, 1983; Kubo, M., Lim, C.S. \& Yamashita, H., The Hosotani Mechanism in Bulk Gauge Theories with an Orbifold Extra Space $S^{1} / Z_{2}$, Mod. Phys. Lett., A17, pp. 2249-2264, 2002.

[9] Csaki, C., Grojean, C., Murayama, H., Pilo, L. \& Terning, J., Gauge Theories on an Interval: Unitary without a Higgs, Phys. Rev., D69, pp. 055006, 2004.

[10] Contino, R., Nomura, Y. \& Pomarol, A., Higgs as a Holographic Pseudo-Goldstone Boson, Nucl. Phys., B671, pp. 148-174, 2003. 
[11] Hall, L.J. \& Nomura, Y., Gauge Coupling Unification from Unified Theories in Higher Dimension, Phys. Rev., D65, pp. 125012, 2002.

[12] Muck, A., Pilaftsis, A. \& Ruckl, R., Minimal Higher-Dimensional Extensions of the Standard Model and Electroweak Observables, Phys. Rev., D65, pp. 085037, 2002.

[13] Schmaltz, M., The Simplest Little Higgs, JHEP, 08, pp. 056, 2004.

[14] Cheng, H.C. \& Low, I., TeV Symmetry and the Little Hierarchy Problem, JHEP, 09, 051, 2003; Cheng, H.C. \& Low, I., Little Hierarchy, Little Higgses, and Little Symmetry, JHEP, 08, pp. 061, 2004.

[15] Kaplan, D.E. \& Schmaltz, M., Little Higgs from a Simple Group, JHEP, 10, pp. 039, 2003.

[16] Schmaltz, M., Physics Beyond the SM Theory: Introducing the Little Higgs, Nucl. Phys. Proc. Supp., 117, pp. 40-49, 2003.

[17] Schmaltz, M. \& Tucker-Smith, D., Little Higgs Review, Ann. Rev. Nucl. Part. Sci., 55, pp. 229-270, 2005.

[18] Coleman, S.R., \& Weinberg, E., Radiative Corrections As The Origin of Spontaneous Symmetry Breaking, Phys. Rev., D7, pp. 1888, 1973.

[19] Scherk, J. \& Schwarz, Spontaneous Breaking of Supersymmetry through Dimensional Reduction, J., Phys. Lett., B82, pp. 60-64, 1979.

[20] Scherk, J. \& Schwarz, J., How to Get Masses from Extra Dimensions, Nucl. Phys., B153, pp. 61-88, 1979.

[21] Hartanto, A. \& Handoko, L.T., Grand Unified Theory based on the SU(6) Symmetry, Phys. Rev., D71, pp. 095013, 2005.

[22] Hartanto, A., Zen, F.P., Kosasih, J.S. \& Handoko, L.T., Near-brane SU(6)Stronglycoupled-Origin Exotic Higgses and Gauge-like Scalar in ScherkSchwarz Breaking of 5-Dimension SU(6) via Double Vacua, Adv. Studies Theor. Phys., 7(8), pp. 375-405, 2013.

[23] Ramond, P., Journeys Beyond the Standard Model, Persus Books, Cambridge, Massachussets, 1999.

[24] McDonald, J., Gauge Singlet Scalars as a Cold Dark Matter, Phys. Rev., D50, pp. 3637, 1994. 